\title{
COLD TOLERANCE OF ENTOMOPATHOGENIC NEMATODES
}

\author{
F. ALI and D.A. WHARTON \\ Department of Zoology, University of Otago, PO Box 56, Dunedin, New Zealand \\ Corresponding author:drfarman@gmail.com
}

\begin{abstract}
Entomopathogenic nematodes in the Steinernematidae and Heterorhabditidae families are lethal parasites of insects. They have great potential as biological control agents since they can be mass produced in vitro. However, their limited shelf life is the major impediment to their large scale commercial application. Attempts to base a storage technology on partial desiccation and anhydrobiosis have met with limited success. The natural capacity of entomopathogenic nematodes for freezing tolerance could be an alternative to be exploited as a first step towards developing a method for their long-term storage. In the present laboratory experiments, the entomopathogenic nematode Steinernema feltiae has shown to be freeze-tolerant if frozen at relatively high sub-zero temperature, with survival near the level of controls. However, slow freezing at high sub-zero temperature $\left(-1^{\circ} \mathrm{C}\right)$ enhanced the nematodes' survival at lower temperatures (down to $-12^{\circ} \mathrm{C}$ ). Freezing of the nematodes was confirmed under the cold stage microscope, although the mechanism of survival in the slow freezing regime was difficult to visualise through cold stage microscopy. This mechanism needs to be investigated further via a different technique such as freeze substitution and electron microscopy. Neither acclimation nor rapid cold hardening improved the survival of nematodes significantly.
\end{abstract}

\section{BIOFUMIGATION WITH BRASSICA SPP. FOR THE CONTROL OF CYLINDROCARPON BLACK FOOT DISEASE OF GRAPEVINES}

\author{
C.M. BLEACH, E.E. JONES and M.V. JASPERS \\ Ecology Department, Faculty of Agriculture and Life Sciences, \\ Lincoln University, PO Box 84, Lincoln 7647, New Zealand \\ Corresponding author: Carolyn.Bleach2@lincolnuni.ac.nz.
}

\begin{abstract}
Green crops of Brassica species incorporated into the soil release volatile isothiocyanates, which are known to suppress pathogenic fungal species. Firstly, crops of mustard (Brassica juncea), rape (B. napus) and oats (Avena sativa) were grown for 5 weeks in a vineyard site previously infested with Cylindrocarpon spp. The crops were cultivated into the soil and the area covered with polythene. After 2 weeks callused cuttings of rootstock 101-104 and 5C were grown for 9 months and infection assessed. Disease incidence in rootstocks 101-14 and 5C was reduced in the mustard treatment by 11 and $43 \%$, respectively. The following year, the site was inoculated with Cylindrocarpon spp. grown on wheat grains. The second experiment used three mustard treatments: mustard meal cultivated into the soil (Trt 1), mustard grown once to flowering with cultivation (Trt 2) and mustard grown twice to flowering with cultivation each time (Trt 3 ). In rootstock $5 \mathrm{C}$ disease incidence was reduced in all treatments by more that $41 \%$ and in rootstock 101-14 disease incidence was reduced in Trt 1 and 3 by 30 and 18\%, respectively. These findings suggest that biofumigation using mustard may be a highly effective method for the control of Cylindrocarpon black foot disease.
\end{abstract}

